\title{
Design and Analytical Evaluation of A High Resistance Sensitivity Bolometer Sensor Based on Plasmonic Metasurface Structure
}

\author{
Abbas Hamouleh-Alipour \\ Lorestan University \\ Ali Mir ( $\square$ mir.a@lu.ac.ir) \\ Lorestan University \\ Ali Farmani \\ Lorestan University
}

\section{Research Article}

Keywords: Analytical, Plasmonic, bolometer, TCR

Posted Date: March 5th, 2021

DOl: https://doi.org/10.21203/rs.3.rs-267998/v1

License: (1) (1) This work is licensed under a Creative Commons Attribution 4.0 International License.

Read Full License

Version of Record: A version of this preprint was published at IEEE Journal of Selected Topics in Quantum Electronics on March 1st, 2022. See the published version at https://doi.org/10.1109/JSTQE.2021.3123049. 


\title{
Design and Analytical Evaluation of a High Resistance Sensitivity Bolometer Sensor Based on Plasmonic Metasurface Structure
}

\author{
Abbas Hamouleh-Alipour ${ }^{1}$, Ali Mir ${ }^{1, *}$, and Ali Farmani ${ }^{1}$ \\ ${ }^{1}$ Department of Electrical and Electronics, Lorestan University, Khorramabad, Iran. \\ *mir.a@lu.ac.ir
}

\begin{abstract}
Bolometer sensors are prominent and excellent choice in technology because they do not need cooling. The trade-off between high sensitivity, fast response time, and strong light absorption is a key important challenge in bolometer sensors. Here, the bolometric effect for a high resistance sensitivity plasmonic sensing of total and profile infusion of radiation is studied for the proposed bolometer sensor based on plasmonic multilayer structure at $26^{\circ} \mathrm{C}$. In the present study, by generating strong coupling condition between incident wave and surface plasmon polaritons (SPPs), a very narrow absorption spectra with high figure of merit (FoM) is achieved. The analytical model and numerical simulation are fulfilled based on the transfer matrix method (TMM) and 3-D finite-difference time-domain (FDTD), respectively. The narrow absorption spectra that generate by strong coupling with SPPs heats the silver thin film that leads to variation in temperature and supports TE surface mode. This temperature change rectifies the resistance of the metal thin film by the bolometric effect. So, optical characteristics of the proposed metasurface bolometer sensor, including quality factor $(\mathrm{Q})$, sensitivity, and figure of merit (FoM) are calculated that Max sensitivity, FoM, and Q are 17.2 RIU-1, 530 and 434.5, respectively. Finally, we analytically simulate the temperature coefficient of resistance (TCR) in terms of wavelength and refractive index of analyte (na) that this resistance change can be monitored by an external electric model. The proposed plasmonic multilayer configuration is a very compact footprint structure that achieved high resistance sensitivity and FoM in comparison with any previous reports. This proposed thermal, optical, and electric plasmonic metasurface bolometer sensor can be used in different applications such as biophysics, biology, and environmental science.
\end{abstract}

\section{Introduction}

Bolometer sensors, known as a device that measure total plasma radiation, can detect electromagnetic (EM) waves by absorbing radiation energy that changes the resistance of the sensor. In other words, proposed sensors are based on absorber structure, which is heated by energy emitted from fusion plasma [1-3]. These kinds of photodetectros have outstanding features for infrared thermal sensing with temperature-dependent resistors due to low cost, ease of manufacturing, and terahertz $(\mathrm{THz})$ detection capability that used for several uncooled sensors, widely [4]. In proposed sensors, using a metallic thin film on the backside of the plasmonic absorber structure, is provided with measurement of the temperature changes [5]. Bolometer sensors are consist of three parts including a thermally isolated freestanding membrane, supporting beams, and a thermistor part [6]. By increasing the absorption coefficient and decreasing the bolometer sensor's thermal conductance, and maximizing the ratio of the temperature coefficient of resistance (TCR) to the normalized noise (1/f), the performance of the bolometer sensors can be improved [7-8]. Enhancement of absorbance with plasmonic perfect absorber can increase responsivity without increasing noise. Originally, high absorbance at mid-infrared and long-wave infrared can be attained based on plasmonic surface plasmon resonance structure with sharp peak absorption and bandwidth depend on resonator $(\mathrm{Q})$. Hence, this structure would improve the fabrication tolerances [9-12]. Usually, high absorbance can be achieved using a plasmonic structure, but the bandwidth is often limited. 
In the past decade, the interest area of research is plasmonic metasurface structure with a very compact footprint that makes a fusion of electronic and optical properties in the form of surface plasmon polaritons (SPPs). This field can be used as superior technology in term of bolometer sensors design [13]. To achieve the best performance, conventional bolometers are designed with multiple layers such as vanadium oxide, $\alpha$-Si, poly-SiGe, and metal that used as thermistors [14]. By considering their small conductivity, often $\mathrm{SiO} 2$ and $\mathrm{Si} 3 \mathrm{~N} 4$ are used as absorbing layers [15-16]. Also, these layers can be replaced with other materials such as metal thin film that reduce the thermal conductance, and these scenarios make a simplify fabrication of device. However, in recent years, several metasurface-based sensing devices have been presented, but no metasurface-based bolometer sensor has been studied until now. Unlike the conventional sensors based on bolometer effect, a metasurface-based bolometer sensor would couple $\mathrm{THz}$ radiation can induce thermal variations rather than an electrical output. In addition to the footprint of the sensor, metasurface-based bolometer sensors would enable to increased sensitivity. Furthermore, the correlation of metasurface was illustrated by the experimental results of a metasurface with size on the order of nanometer [17-23]. The artificial sheet with very narrow thickness is used as a material for metasurface sensors. In other words, metasurfaces can be either structured or unstructured with subwavelength-scaled patterns in the $\mathrm{Z}$ dimension which leads to a high phase sensitive system [24]. Furthermore, cooling and heating actions of metasurface sensors have been experimentally demonstrated, recently [25].

According to the aforementioned details, we propose a high resistance sensitivity bolometer sensor based on the metasurface multilayer structure. We numerically realize perfect absorber sensors using the FDTD method and TMM as an analytical model. Results show a good operation in near-infrared for the presented sensor. Also, high sensitivity, high quality factor, very compact footprint and, TCR are achieved in comparison with recent works in the field of bolometer sensors.

The presented paper is organized as follows: after the introduction in part I, theory, model, and analytical consideration are explained in part II. Section III introduces the results and discussion, and eventually, the paper is concluded in part IV.
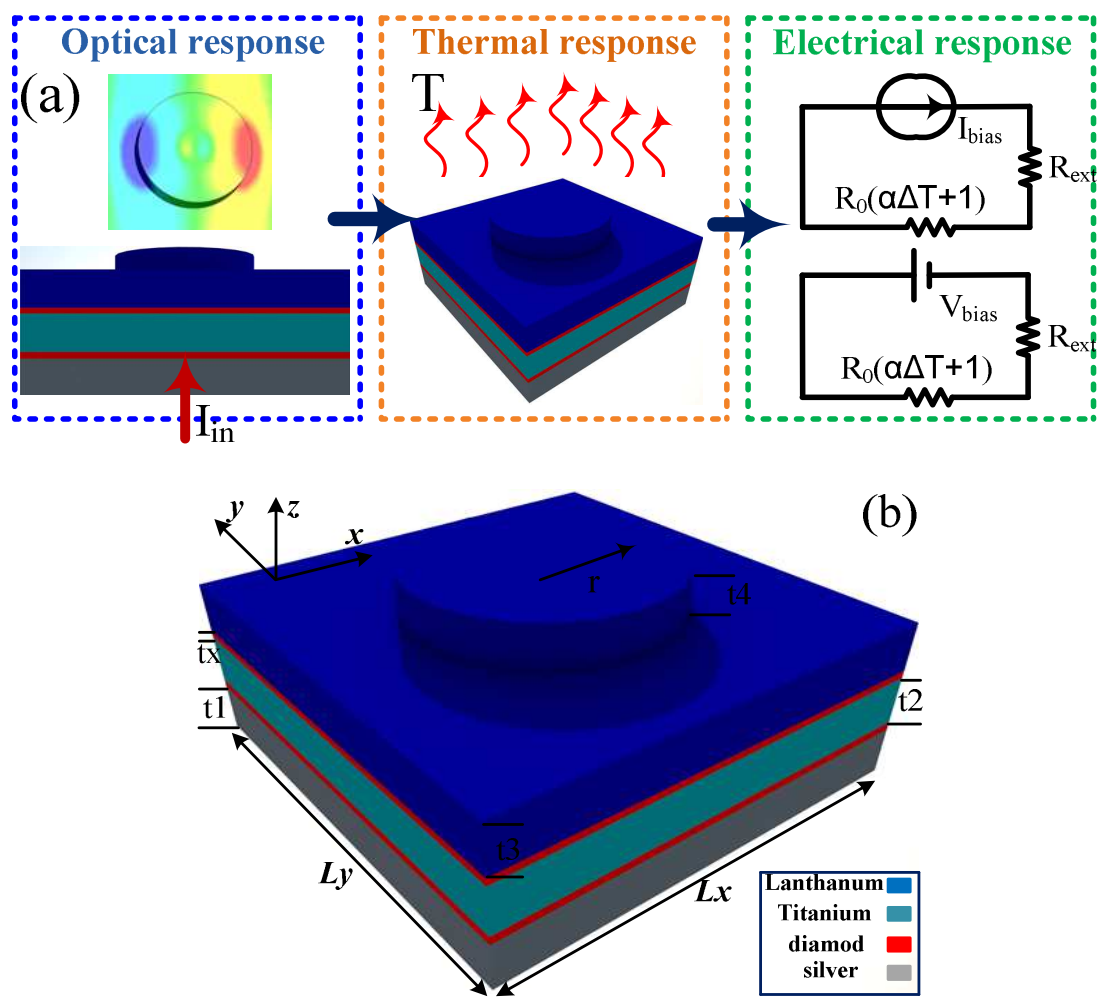

Fig. 1. (a) Schematic representation of the physical phenomena including optical, thermal and electronic response and (b) The 3D-views schematic of the proposed structure. 


\section{THEORY, MODEL AND ANALYTICAL CONSIDERATION}

\section{A. Sensor Configuration and Material Model}

Here, the configuration and layers of the proposed sensor are introduced. After this, the analytical consideration of the sensor is presented. As mentioned in the previous part, increasing the absorption spectra leads to increase the temperature of metal thin layer that consequently changes its electric resistance based on bolometer effect. Also based on an external circuit biased with a voltage or current source, we can measure the resistance change as voltage change. All proposed procure summarize in Fig. 1(a). The proposed plasmonic metasurface bolometer sensor is depicted in Fig. 1(b) where, we have chosen the FDTD model to extract numerical results. As can be seen in Fig. 1(b), the sensor consists of five layers as a multilayer structure. The layers with the refractive index and lattice constant are reported in Table. I. It can be deduced from Table. I that the presented sensor can be fabricated without any problems based on the lattice constant of each layer. As can be seen in the 3D view of the sensor (Fig. 1(b)), the length (Lx) and width (Ly) of the structure are in optimum condition and $600 \mathrm{~nm}$ and $600 \mathrm{~nm}$. It can be stated that the overall size of the proposed plasmonic metasurface bolometer sensor is optimized based on particle swarm optimization (PSO) algorithm by using the CST package, which evaluates the interaction between EM wave and thermal domain. PSO is a computational method that optimizes a problem by iteratively trying to improve a candidate solution with regard to a given measure of quality. The structure consists of multilayer that are arranged as silver-diamond-titanium-diamond-lanthanum (analyte). The bolometer sensor has a very compact footprint that the best candidate to be used in nanoelectronic devices. Silver is usually modeled by frequency-dependent Drude's model that consists of two terms including, real $(\varepsilon \operatorname{Re})$ and imaginary $(\varepsilon \mathrm{Im})$ parts, which is expressed as [26]:

$$
\begin{aligned}
& \varepsilon(\omega)=\varepsilon_{\mathrm{Re}}(\omega)+i \varepsilon_{\mathrm{Im}}(\omega) \\
& =1-\frac{\omega_{p}^{2}}{\omega\left(\omega+i \gamma_{c}\right)}=\left(1-\frac{\omega_{p}^{2}}{\omega^{2}+\gamma_{c}^{2}}\right)+i\left(\frac{\omega_{p}^{2} \gamma_{c}^{2}}{\omega\left(\omega_{p}^{2}+\gamma_{c}^{2}\right)}\right)
\end{aligned}
$$

In the above equation, $\omega$ is the angular frequency of the incident wave, damping rate is $\gamma_{c}=2.73 \times 10^{13}$ rad. $\mathrm{s}^{-1}$ and bulk plasmon frequency is $\omega_{\mathrm{p}}=1.38 \times 10^{16}$ rads- 1 . Also, to model the lanthanum or analyte, we used the Sellmaier equation that explains by [27]:

$$
n^{2}-1=\frac{1.85744228 \lambda^{2}}{\lambda^{2}-0.00982397191}+\frac{0.294098129 \lambda^{2}}{\lambda^{2}-0.0384309138}+\frac{1.1665417 \lambda^{2}}{\lambda^{2}-89.3984634}
$$

where $\mathrm{n}$ is the refractive index, and $\lambda$ is the wavelength of the incident light. Table I present the refractive index, lattice constant and thickness of the sensor layers. 
Table I: Refractive index, lattice constant and thickness of the proposed sensor layers

\begin{tabular}{cccc}
\hline material & Refractive index & Lattice constant & Thickness $(\mathrm{nm})$ \\
\hline silver & Drude model & $4.0853 \mathrm{~A}$ & $\mathrm{t} 1=100$ \\
Titanium & $1.81(\varepsilon=3.2761)$ & $2.9805 \mathrm{~A}$ & $\mathrm{t} 2=150$ \\
Lanthanum & Sellmaier & $3.772 \mathrm{~A}$ & $\mathrm{t} 3+\mathrm{t} 4=285$ \\
Diamond & na $=4.2$ & $3.5 \mathrm{~A}$ & $\mathrm{t} \mathrm{x}=5$ \\
\hline
\end{tabular}

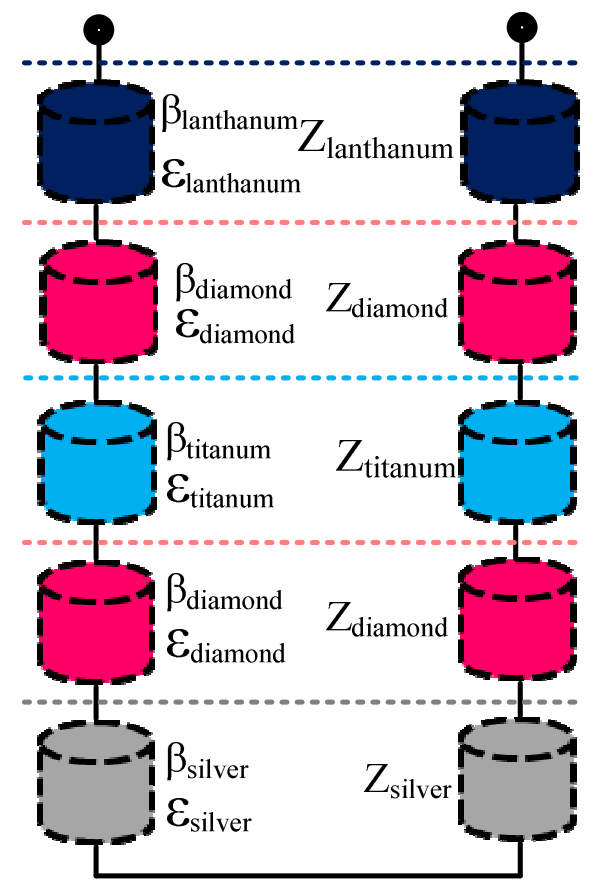

Fig. 2. The distributed circuit model of the structure in TM polarization.

\section{B. Analytical Model}

Now, the transfer matrix model (TMM) is explained to demonstrate a distributed circuit model (DCM) as an analytical model for the proposed bolometer metasurface multilayer sensor. Generally, each layer is modelled by a transmission line with an impedance of $\mathrm{Zi}$ and propagation constant of $\beta \mathrm{i}$. Impedance and propagation constant are related to polarization and angle of the incident light. Fig. 2, depicts the distributed circuit model of the proposed bolometer sensor. As shown in this figure, each layer is a model with characteristics impedance $(Z)$, propagation constant $(\beta)$ and, electric permittivity, $\varepsilon$. In the proposed Transfer matrix model, each layer with a specific transmission line is linked to each other with a $2 \times 2$ transfer matrix. So, by using the TM of each layer, the TM model of the proposed sensor can be determined. Consequently, the TM model of the bolometer effect of the structure introduced above can be stated as [28]:

$$
\left(\begin{array}{ll}
A_{11} & A_{12} \\
A_{21} & A_{22}
\end{array}\right)=N_{\text {silver }} \times N_{\text {diamond }} \times N_{\text {titanum }} \times N_{\text {diamond }} \times N_{\text {lantanum }}
$$


where, $N_{\text {silver }}, N_{\text {dia }}, N_{\text {titanum }}$ and $N_{\text {lantanum }}$ are the transfer matrix of the silver, diamond, titanium, and lanthanum, respectively. These matrixes can be defined as:

$$
\begin{aligned}
& N_{\text {silver }}=\left(\begin{array}{cc}
\cos \left(\beta_{\text {silver }} t_{1}\right) & j Z_{0} \sin \left(\beta_{\text {silver }} t_{1}\right) \\
\frac{j}{z_{\text {silver }}} \sin \left(\beta_{\text {siver }} t_{1}\right) & \cos \left(\beta_{\text {silver }} t_{1}\right)
\end{array}\right) \\
& N_{\text {diamond }}=\left(\begin{array}{cc}
\cos \left(\beta_{\text {diamond }} t_{2}\right) & j Z_{0} \sin \left(\beta_{\text {diamond }} t_{2}\right) \\
\frac{j}{z_{\text {dimmox }}} \sin \left(\beta_{\text {diamond }} t_{2}\right) & \cos \left(\beta_{\text {diamond }} t_{2}\right)
\end{array}\right) \\
& N_{\text {titanum }}=\left(\begin{array}{cc}
\cos \left(\beta_{\text {titanum }} t_{3}\right) & j Z_{0} \sin \left(\beta_{\text {titanum }} t_{3}\right) \\
\frac{j}{Z_{\text {tiamm }}} \sin \left(\beta_{\text {titanum }} t_{3}\right) & \cos \left(\beta_{\text {titanum }} t_{3}\right)
\end{array}\right) \\
& N_{\text {lantanum }}=\left(\begin{array}{cc}
\cos \left(\beta_{\text {lantanum }} t_{4}\right) & j Z_{0} \sin \left(\beta_{\text {lantanum }} t_{4}\right) \\
\frac{j}{z_{\text {lanturm }}} \sin \left(\beta_{\text {lantanum }} t_{4}\right) & \cos \left(\beta_{\text {lantanum }} t_{4}\right)
\end{array}\right)
\end{aligned}
$$

where $\beta$ is the propagation constant of TM polarization waves that can be determined in each region as follows:

$$
\begin{aligned}
& \beta_{\text {silver }}=t_{1} \sqrt{\varepsilon_{\text {silver }}-n_{1}^{2} \sin ^{2} \theta} \\
& \beta_{\text {titanum }}=t_{3} \sqrt{\varepsilon_{\text {titanum }}-n_{1}^{2} \sin ^{2} \theta} \\
& \beta_{\text {lantanum }}=t_{4} \sqrt{\varepsilon_{\text {lantanum }}-n_{1}^{2} \sin ^{2} \theta}
\end{aligned}
$$

So, the reflection spectra of the proposed filter can be calculated based on the overall transfer matrix element as follow:

$$
R=\frac{\kappa\left(A_{22}-A_{11}\right)-\left(\kappa^{2} A_{22}-A_{11}\right)}{\kappa\left(A_{22}+A_{11}\right)-\left(\kappa^{2} A_{22}+A_{11}\right)}
$$

In the above equation $\kappa=\beta_{0} / \kappa_{0}$ that $\kappa_{0}$ is the wave-number of free space. In the next section of the proposed manuscript, we assume that incident light has wavelengths from $\lambda=1000 \mathrm{~nm}$ to $\lambda=1200 \mathrm{~nm}$, and then calculate the absorption, reflectance, and electrical filed distribution. 


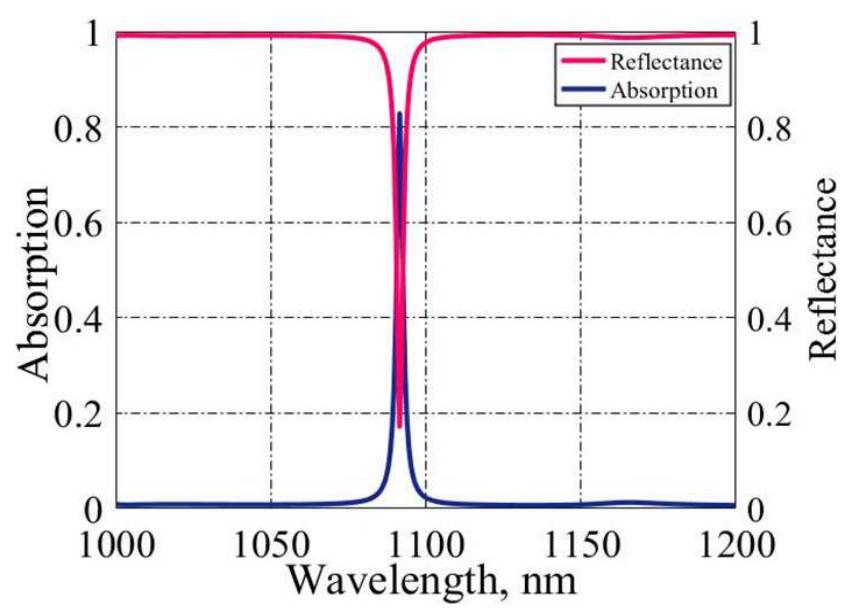

Fig. 3. The absorption and reflectance spectra of the proposed senor.

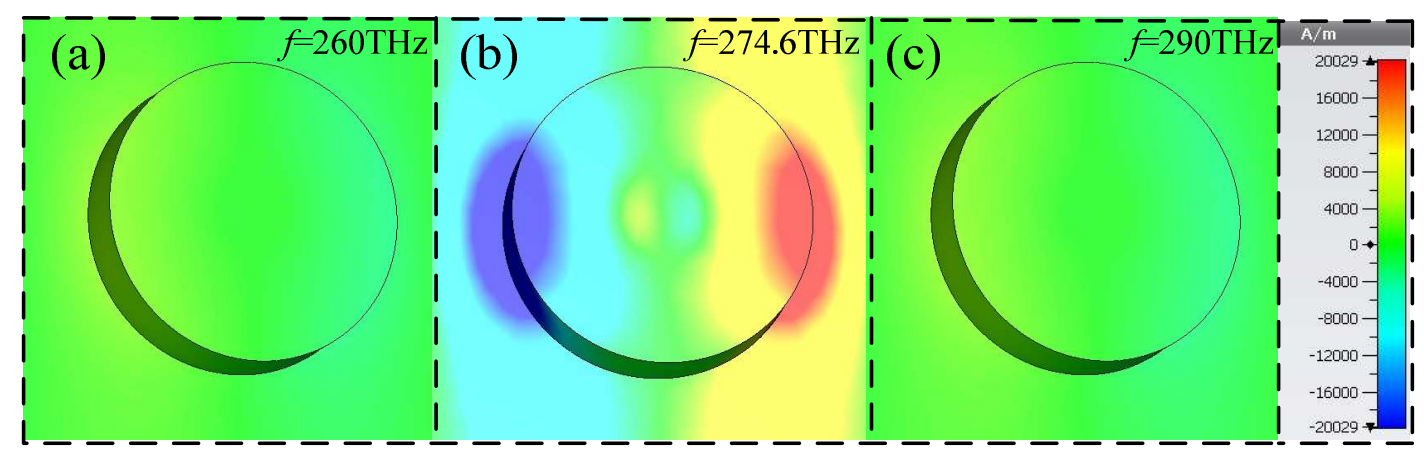

Fig. 4. Magnetic field distribution at different frequencies (a) $f=260 \mathrm{THz}$, (b) $f=274.6 \mathrm{THz}$ and (c) $f=290$ THz.

\section{RESULTS AND DISCUSSION}

In the results and discussion part, we numerically calculate the results of the bolometer sensor depicted in section II. Physically, the perfect absorption appears when a very narrow spectral resonance interferes with a broadband background, coherently. In the presented sensor, the narrow peak is caused by an SPR appearing at the metal/dielectric interface, and the broadband spectral background is produced by the radiation scattered from the grating structure, that is illuminated by the same source. The equation of the total absorption spectrum is explained as below [29]:

$$
\frac{2 \pi m}{P}=\frac{\omega}{c} \sqrt{\frac{\varepsilon_{m} \varepsilon_{e f f}}{\varepsilon_{m}+\varepsilon_{e f f}}}
$$

where $P$ is the grating period, m represents the diffraction order, $c$ is the speed of light, $\varepsilon_{\mathrm{m}}$ is the real part of the dielectric permittivity of the metal, and $\varepsilon_{\text {eff }}$ is the effective dielectric permittivity of the effective media where the plasmon propagates. In this section, we try to increase the temperature of the metal layer. To do it, we must enhance absorption at the metal layer, which in combination with the nanostructure. We 
consider as an almost-perfect absorber. The absorption spectra of the proposed sensor are illustrated in Fig. 3. As can be seen in this figure, the maximum absorption is 0.85 at $1091.5 \mathrm{~nm}$ wavelength. The resonant wavelength can be tuned by adjusting the periodicity $\mathrm{P}$ in Equation 12 . The magnetic field distribution $(\mathrm{Hz})$ of the proposed bolometer sensor for different frequencies is shown in Fig. 4. It is clear that for $\mathrm{f}=274.6 \mathrm{THz}$, strong coupling between light and surface of the proposed bolometer sensor has occurred. The dipole filed in the surrounded region of the circle at bottom of the structure shows this strong coupling. Also, at other frequencies such as $f=260$ and $f=290 \mathrm{THz}$, no coupling has appeared (Fig. 4(a) and (b)).

To study the electric resistivity of the materials, we explain the temperature coefficient of resistance (TCR) or $\alpha$ equation as bellow [29]:

$$
R=R_{0}\left[1+\alpha\left(T-T_{0}\right)\right]
$$

The value of TCR for silver is $\alpha=0.0036 \mathrm{k}^{-1}$. Also, the temperature variation is related to the absorbed power and can be calculated as [29]:

$$
\nabla .(k \nabla T)=Q
$$

where $k$ is the thermal conductivity and $Q$ is the heat power density, which is related to the EM power absorbed by the metal layer. Fig. 5 shows the maximum temperature versus of the incident EM wavelength. As can be seen, the maximum response of temperature appears at $\lambda=1091.5 \mathrm{~nm}$. For the proposed case, the temperature reaches 295.25.

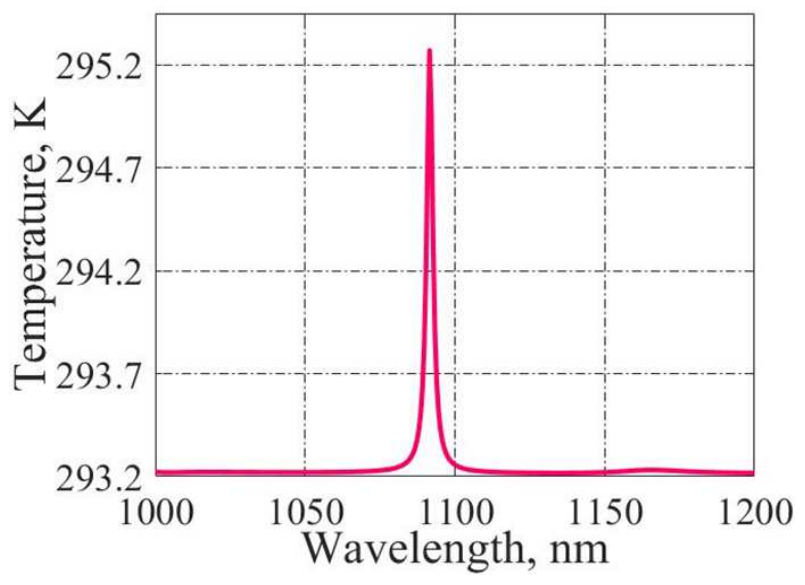

Fig. 5. Maximum temperature versus the incident EM wavelength

To investigate the operation of the proposed sensor as a bolometer sensor, the metal thin film in the sensor's configuration is connected to an external electric circuit such as a voltage divider. The output of the bolometer effect of the sensor is dependent on the resistance of the silver metal thin film, R0. Based on the bellow equation, it is clear that $\mathrm{R}_{0}$ is dependent on the geometry [29]:

$R_{0}=\rho_{0} L /\left(t_{m} \times w\right)$

where $\rho_{0}$ is the resistivity of the metal thin film at room temperature, $L$ is the length, tm is the transversal section as the product of thickness and $w$ is transversal width. Fig. 6 is depicted the spectra resistance 
variation of the proposed bolometer sensor. As can be seen, it is obvious that by increasing the refractive index of the analyte, the maximum of $\Delta R$ is enhanced to the higher wavelengths. So, we show that the maximum of $\Delta R$ moves from $\lambda_{1}=1091.5 \mathrm{~nm}$ to $\lambda_{2}=1220 \mathrm{~nm}$.

The variation in resistance versus the refractive index of the analyte, na, is shown in Fig. 7 (fitting and without fitting spectra). For a fixed value of maximum resonance like $\lambda=1091.5 \mathrm{~nm}$, enhancement in na causes in decrease of $\Delta \mathrm{R}$. As can be seen in Fig. 7, it is clear that the response is symmetric when the refractive index is the lower value. The bridge between Optical and electrical responses is based on an external biasing source that showed the practical performance of the bolometer sensor. The voltage change $\Delta V_{\text {out }}$ in versus of resistance change $\Delta \mathrm{R}$ that depending on the biasing voltage or current is given as, respectively [29]:

$$
\begin{aligned}
& \Delta V_{\text {out }}=V_{\text {bias }} \frac{R_{\text {ext }}}{\left(R_{\text {ext }}+R_{0}\right)^{2}} \Delta R \\
& \Delta V_{\text {out }}=I_{\text {bias }} \Delta R
\end{aligned}
$$

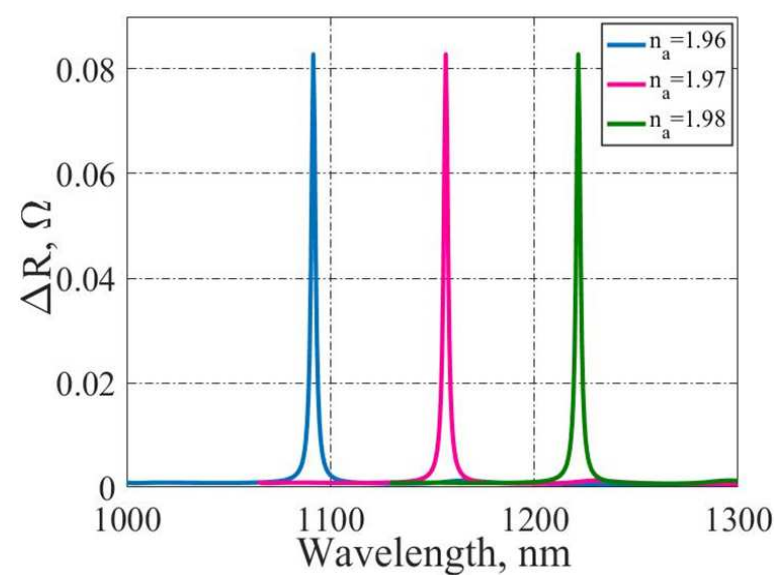

Fig. 6. The spectra of the maximum $\Delta R$ versus of the incident wavelength at different refractive index of the analyte.

In the above mentioned equations, the signal $\Delta V_{\text {out }}$ is related to $\Delta R$, proportionally. Based on these equations, we can define the sensitivity $\left(S_{\mathrm{B}}\right)$, and figure of merit (FoM) as bellow [29]:

$$
S_{B}=\frac{\partial}{\partial \mathrm{n}_{\mathrm{a}}}(\Delta R)
$$

$$
F o M=\frac{S_{\mathrm{B}}}{\Delta R}
$$




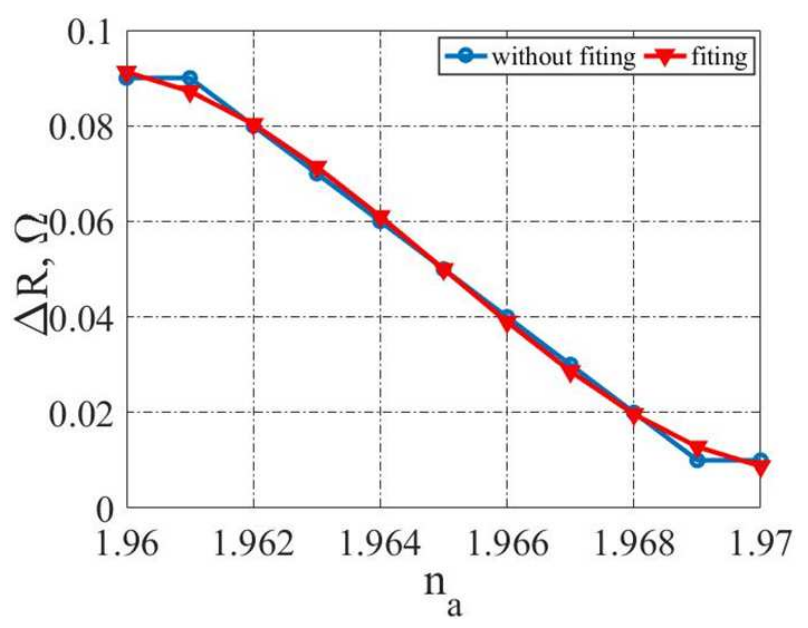

Fig. 7. Variation of the resistance versus of refractive index of analyte, $n_{\mathrm{a}}$, (fitting and without fitting spectra).

To demonstrate the operation of the proposed bolometer sensor, the sensitivity based on the bolometric effect is calculated at $\lambda=1091.5 \mathrm{~nm}$ as a function of the refractive index of the analyte. Fig. 8 shows the sensitivity vs. refractive index variations from 1.96 to 1.97 (fitting and without fitting spectra). Based on Eq. 17, sensitivity of the bolometer sensor is achieved. This parameter is 17.2 for $\lambda=1091.5 \mathrm{~nm}$ at 1.964 refractive indexes (na). Also, based on this parameter and Eq. 18, the FoM of the sensor is calculated. In the proposed sensor, the maximum FoM is 530 1/RIU, which shows a good performance in comparison with other bolometer sensors. So, for this purpose, the FoM of the sensor is depicted in Fig. 9 (fitting and without fitting spectra).

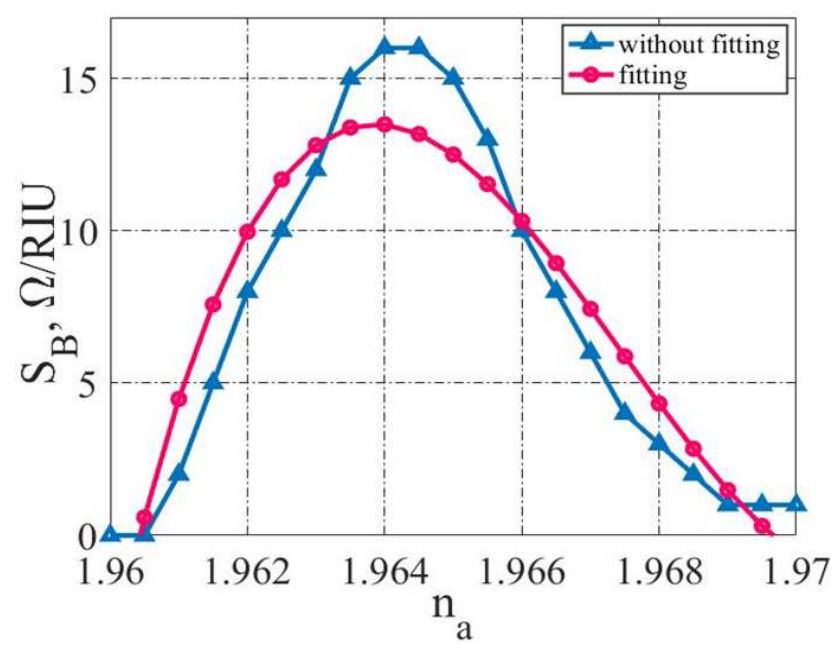

Fig. 8. Sensitivity of the proposed senor versus the refractive index variations of the analyte (fitting and without fitting spectra). 


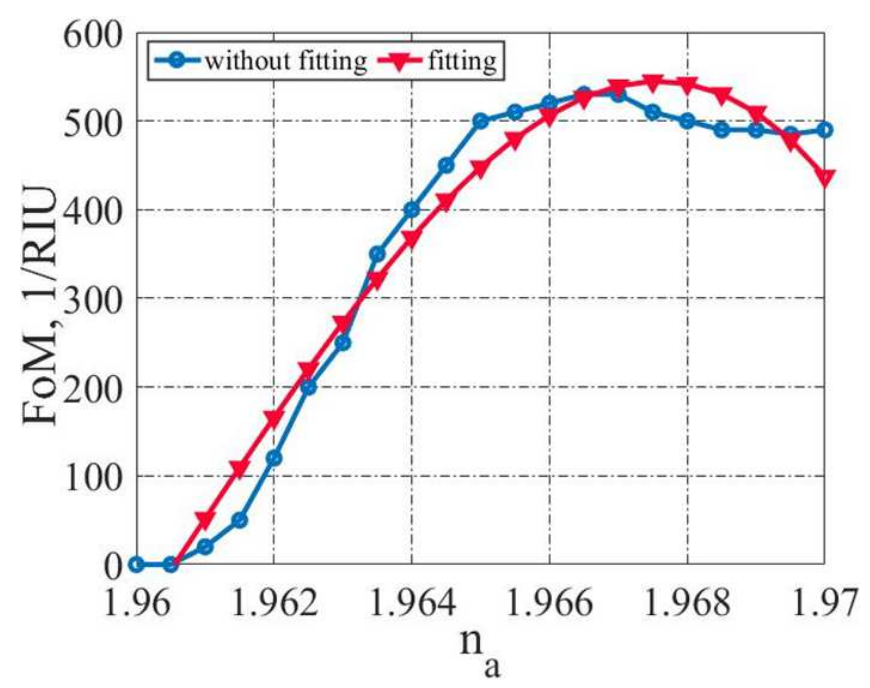

Fig. 9. FoM of the proposed bolometer sensor as a function of the refractive index variation (with fitting and without fitting spectra).

\section{CONCLUSION}

In conclusion, we study a bolometer mechanism of a high resistance sensitivity sensor based on a plasmonic metasurface multilayer structure at room temperature. Using strong interaction between SPPs and incident light, a very narrow absorption spectra related to heat power is achieved. Based on the proposed configuration, the transfer matrix method of the sensor is presented. Also, by considering the FDTD model, we simulate the bolometer effect of the sensor with high sensitivity and FoM. Temperature change in the silver thin film leads to a narrow absorption that rectifies the resistance of the metal thin film by the bolometric effect. The optical characteristics including, $Q$ factor, EM fields, sensitivity, and FoM are analytically and numerically calculated. By using the extracted model of the sensor, the temperature coefficient of resistance (TCR) versus the incident wavelengths and refractive index of the analyte $\left(n_{\mathrm{a}}\right)$ is simulated. Considering abovementioned performance of the proposed sensor, the overall size of the sensor is very compact as $600 \times 600 \times 545 \mathrm{~nm}^{3}$ with high resistance sensitivity and FoM that is comparable to pervious works. The presented thermal, optical and electrical bolometer sensor can be used for different applications such as bio-physics, biology and, etc. performance.

\section{References}

[1] A. Varpula, K. Tappura, J. Tiira, K. Grigoras, J. Ahopelto, and M. Prunnila. "Nano-Thermoelectric Infrared Bolometer." arXiv preprint arXiv: 1912.12868 (2019).

[2] R. S. Saxena, , R. K. Bhan, P. Singh Rana, A. K. Vishwakarma, A. Aggarwal, K. Khurana, and S. Gupta. "Study of performance degradation in titanium microbolometer IR detectors due to elevated heating." Infrared Physics \& Technology, 54, no. 4 (2011): 343-352.

[3] A. Banerjee, H. Satoh, D. Elamaran, Y. Sharma, N. Hiromoto, and H. Inokawa. "Optimization of narrow width effect on titanium thermistor in uncooled antenna-coupled terahertz microbolometer." Japanese Journal of Applied Physics, 57, no. 4S (2018): 04FC09. 
[4] S. Abbasi, A. Shafique, O. Ceylan, C. B. Kaynak, M. Kaynak, and Y. Gurbuz. "A test platform for the noise characterization of SiGe microbolometer ROICs." IEEE Sensors Journal, 18, no. 15 (2018): 6217-6223.

[5] Y. Pan, G. Tagliabue, H. Eghlidi, C. Höller, S. Dröscher, G. Hong, and D. Poulikakos. "A rapid response thin-film plasmonic-thermoelectric light detector." Scientific reports, 6 (2016): 37564.

[6] C. V. Hoof, and P. D. Moor. "PolySiGe uncooled microbolometers for thermal IR detection." (2002): 449-477.

[7] D. Elamaran, Y. Suzuki, H. Satoh, A. Banerjee, N. Hiromoto, and H. Inokawa. "Performance Comparison of SOI-Based Temperature Sensors for Room-Temperature Terahertz Antenna-Coupled Bolometers: MOSFET, PN Junction Diode and Resistor." Micromachines, 11, no. 8 (2020): 718.

[8] M. V. S. Ramakrishna, G. Karunasiri, P. Neuzil, U. Sridhar, and W. J. Zeng. "Highly sensitive infrared temperature sensor using self-heating compensated microbolometers." Sensors and Actuators A: Physical, 79, no. 2 (2000): 122-127.

[9] D. Wu, Y. Liu, R. Li, L. Chen, R. Ma, C. Liu, and H. Ye. "Infrared perfect ultra-narrow band absorber as plasmonic sensor." Nanoscale research letters, 11, no. 1 (2016): 1-9.

[10] P. Mandal "Plasmonic perfect absorber for refractive index sensing and SERS." Plasmonics, 11, no. 1 (2016): 223-229.

[11] D. Wu, R. Li, Y. Liu, Z. Yu, L. Yu, L. Chen, C. Liu, R. Ma, and H. Ye. "Ultra-narrow band perfect absorber and its application as plasmonic sensor in the visible region." Nanoscale research letters, $\mathbf{1 2}$, no. 1 (2017): 1-11.

[12] M. Janneh, A. D. Marcellis, E. Palange, A. T. Tenggara, and D. Byun. "Design of a metasurfacebased dual-band Terahertz perfect absorber with very high Q-factors for sensing applications." Optics Communications, 416 (2018): 152-159.

[13] M. I. Stockman, , K. Kneipp, S. I. Bozhevolnyi, S. Saha, A. Dutta, J. Ndukaife, N. Kinsey and et al. "Roadmap on plasmonics." Journal of Optics, 20, no. 4 (2018): 043001.

[14] H-Y. Lee, C-L. Wu, C-H. Kao, C-T. Lee, S-F. Tang, W-J. Lin, H-C. Chen, and J-C. Lin. "Investigated performance of uncooled tantalum-doped VOx floating-type microbolometers." Applied Surface Science, 354 (2015): 106-109.

[15] L. Gao, F. Lemarchand, and M. Lequime. "Refractive index determination of $\mathrm{SiO} 2$ layer in the UV/Vis/NIR range: spectrophotometric reverse engineering on single and bi-layer designs." Journal of the European Optical Society-Rapid publications, 8 (2013).

[16] S. Ferré, A. Peinado, E. Garcia-Caurel, V. Trinité, M. Carras, and R. Ferreira. "Comparative study of $\mathrm{SiO} 2, \mathrm{Si} 3 \mathrm{~N} 4$ and $\mathrm{TiO} 2$ thin films as passivation layers for quantum cascade lasers." Optics express, 24, no. 21 (2016): 24032-24044.

[17] Y. Zhou, I. I. Kravchenko, H. Wang, H. Zheng, G. Gu, and J. Valentine. "Multifunctional metaoptics based on bilayer metasurfaces." Light: Science \& Applications, 8, no. 1 (2019): 1-9.

[18] Z. Liu, , Y. Xu, C-Y. Ji, S. Chen, X. Li, X. Zhang, Y. Yao, and J. Li. "Fano-Enhanced Circular Dichroism in Deformable Stereo Metasurfaces." Advanced Materials, 32, no. 8 (2020): 1907077. 
[19] A. Alipour, A. Farmani, and A. Mir. "High sensitivity and tunable nanoscale sensor based on plasmon-induced transparency in plasmonic metasurface." IEEE Sensors Journal, 18, no. 17 (2018): 7047-7054.

[20] A H. Alipour, and A. Mir. "Design and simulation of a high-selective plasmon-induced reflectance in coupled dielectric-metal-dielectric nano-structure for senor devices and slow light propagation." Plasmonics 14, no. 2 (2019): 511-521.

[21] A. Alipour, A. Mir, and A. Farmani. "Ultra-high-sensitivity and tunable dual-band perfect absorber as a plasmonic sensor." Optics \& Laser Technology, 127 (2020): 106201.

[22] A. Alipour, A. Farmani, and A. Mir. "SiO 2-silver Metasurface architectures for ultrasensitive and tunable Plasmonic biosensing." Plasmonics, 15, no. 6 (2020): 1935-1942.

[23] A. J. Golfazani, A. Alipour, M. Bakhshipour, A. Farmani, and A. Mir. "Analytical and Numerical Models of a Highly Sensitive MDM Plasmonic Nano-structure in Near-infrared Range." Plasmonics, (2020): 1-6.

[24] Y. Lee, S-J. Kim, H. Park, and B. Lee. "Metamaterials and metasurfaces for sensor applications." Sensors, 17, no. 8 (2017): 1726.

[25] J-Y. Jung, K. Song, J-H. Choi, J. Lee, D-G. Choi, J-H. Jeong, and D. P. Neikirk. "Infrared broadband metasurface absorber for reducing the thermal mass of a microbolometer." Scientific Reports, 7, no. 1 (2017): $1-8$.

[26] Z. Wei, X. Li, N. Zhong and et all "Analogue Electromagnetically Induced Transparency Based on Low-loss Metamaterial and its Application in Nano sensor and Slow-light Device." Plasmonics, 12, 641-647, (2017).

[27] J. Mistrik, et al. "Optical properties of electronic materials: fundamentals and characterization." Springer Handbook of Electronic and Photonic Materials. Springer, Cham, 2017. 1-1.

[28] A. Farmani, M. Miri, and M. H. Sheikhi. "Analytical modelling of highly tunable giant lateral shift in total reflection of light beams from a graphene containing structure." Optics Communications, 391 (2017), 68-76.

[29] M. H.Elshorbagy, A. Cuadrado, J. A. Gómez-Pedrero, and J. Alda. "Opto-Electronic Refractometric Sensor Based on Surface Plasmon Resonances and the Bolometric Effect." Applied Sciences, 10, no. 4 (2020): 1211. 
Figures
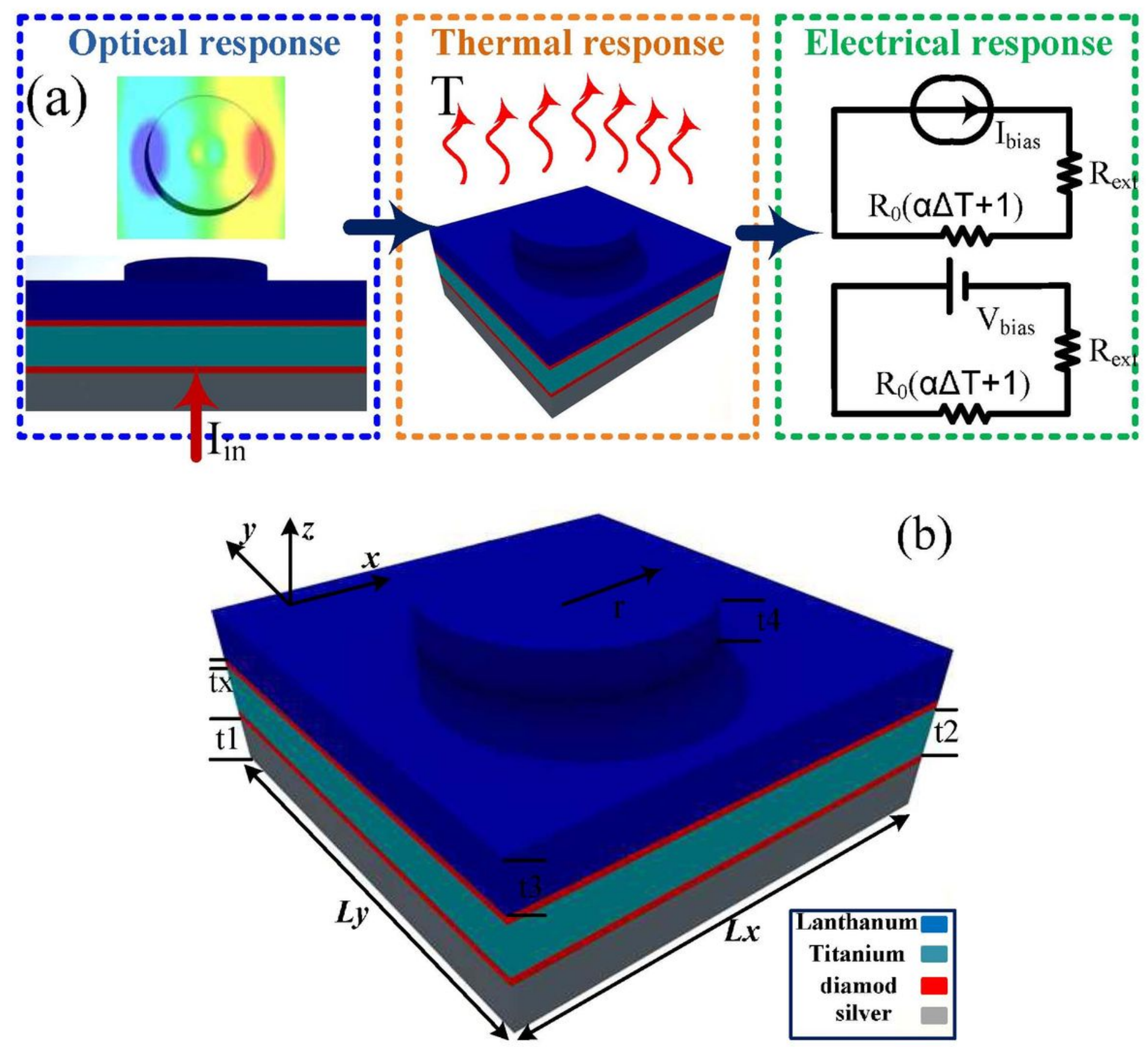

Figure 1

(a) Schematic representation of the physical phenomena including optical, thermal and electronic response and (b) The 3D-views schematic of the proposed structure. 


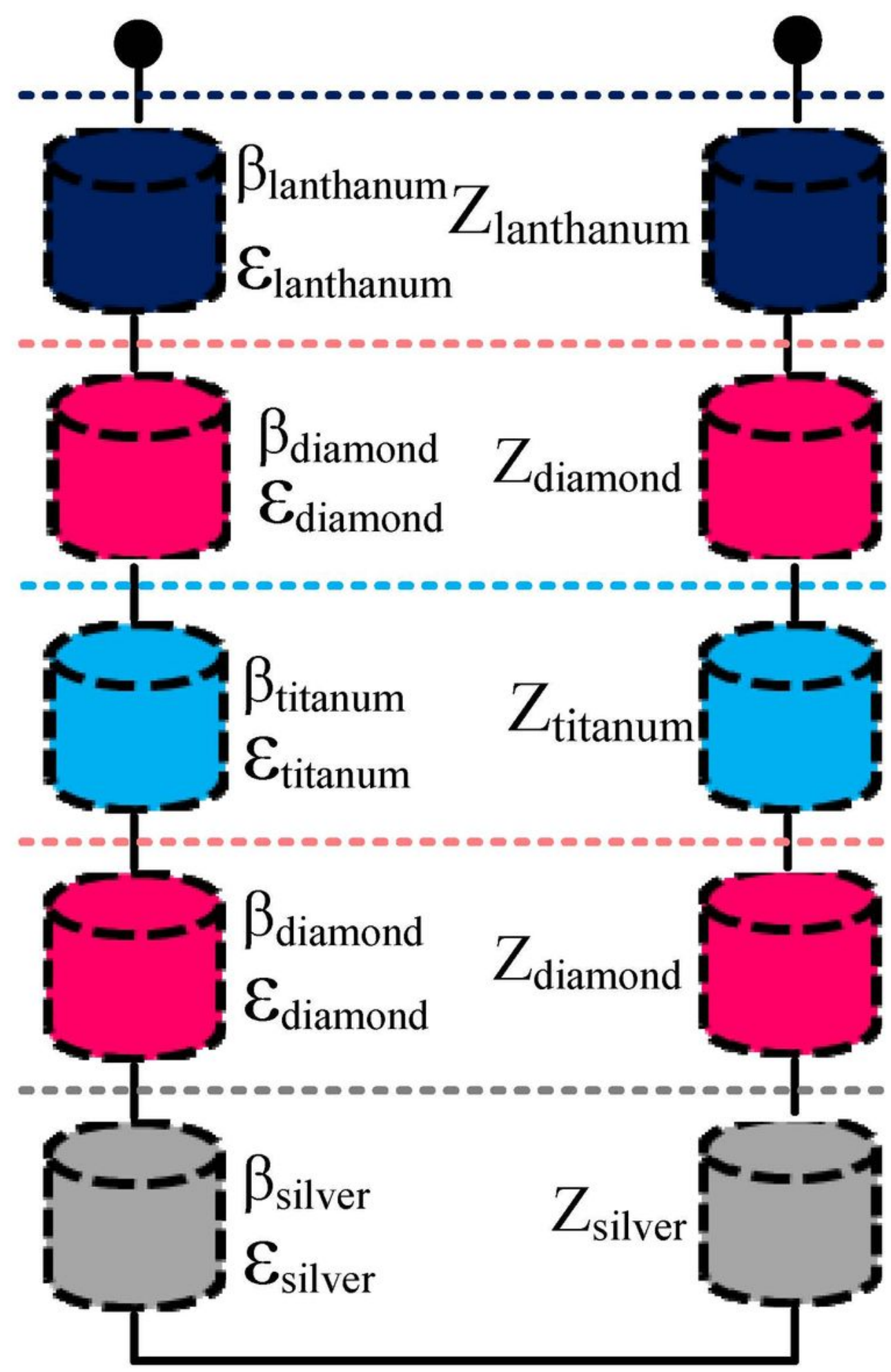

Figure 2

The distributed circuit model of the structure in TM polarization. 


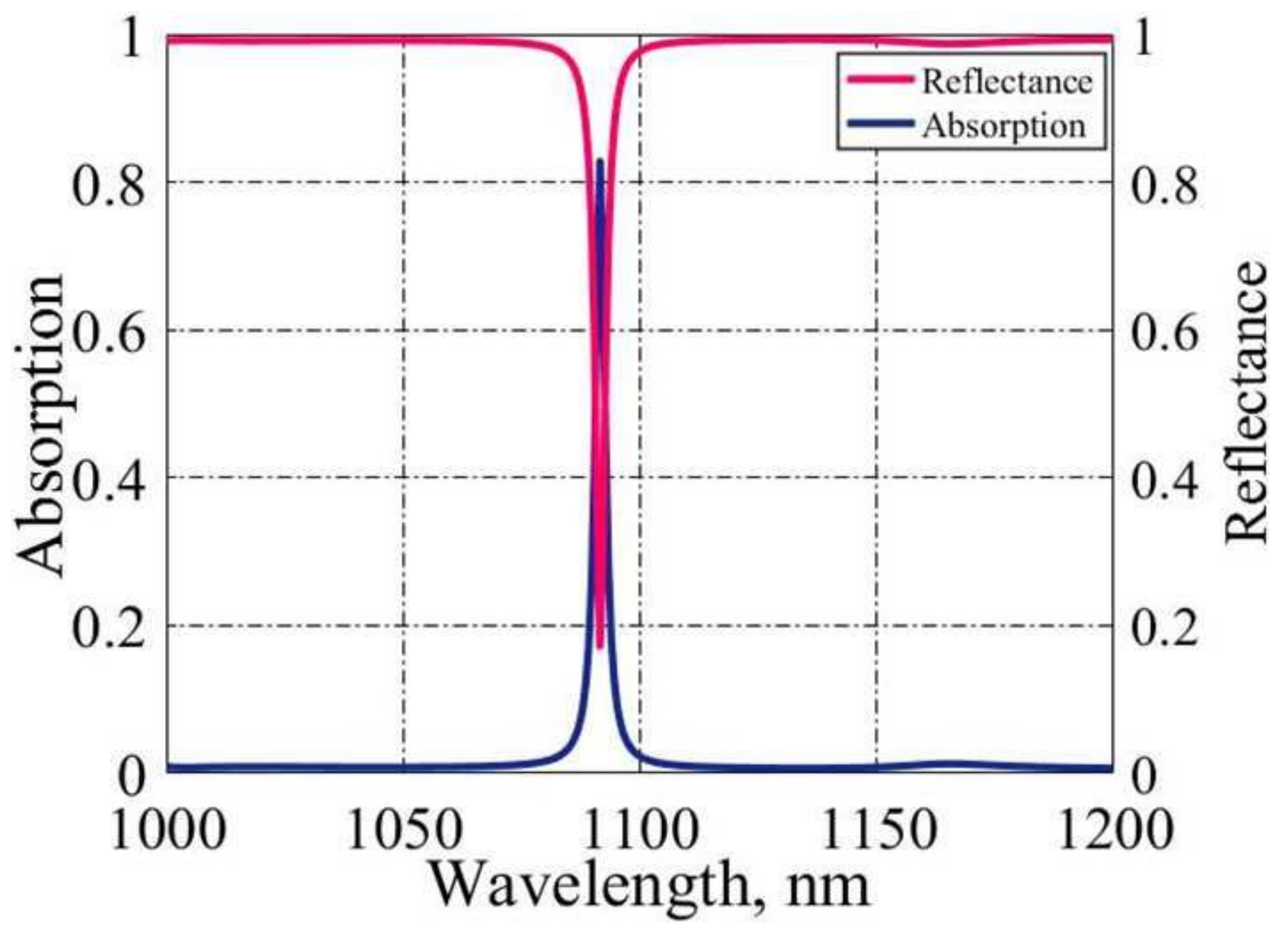

Figure 3

The absorption and reflectance spectra of the proposed senor.

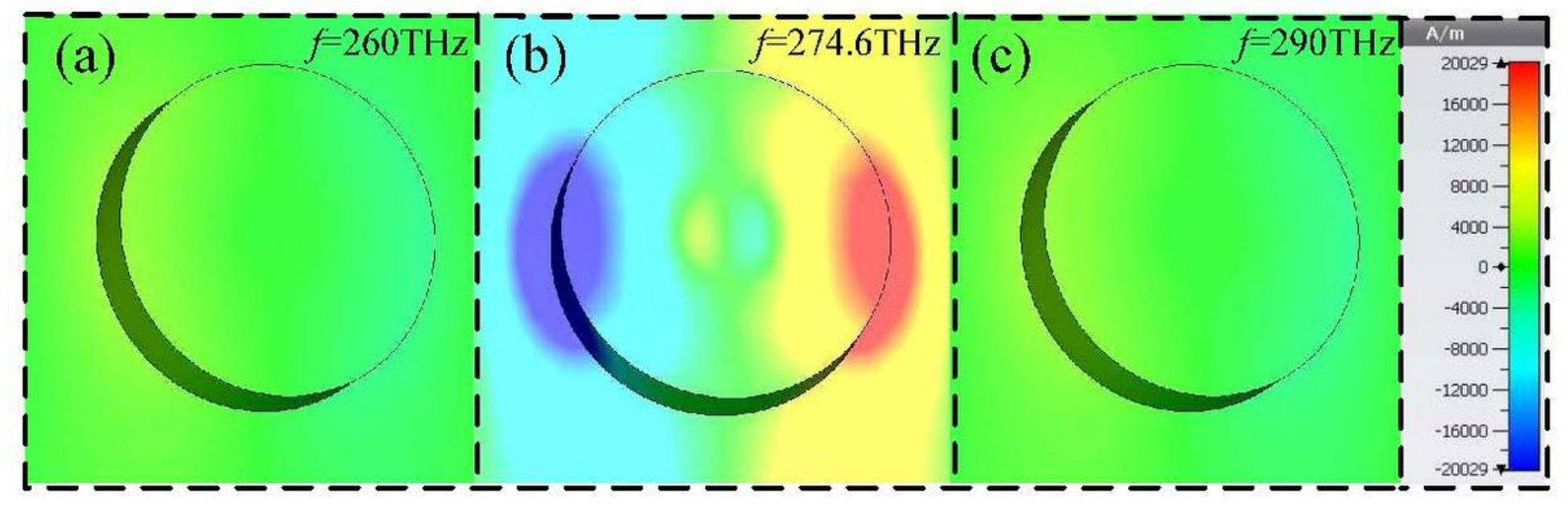

Figure 4

Magnetic field distribution at different frequencies (a) $f=260 \mathrm{THz}$, (b) $f=274.6 \mathrm{THz}$ and (c) $f=290 \mathrm{THz}$. 


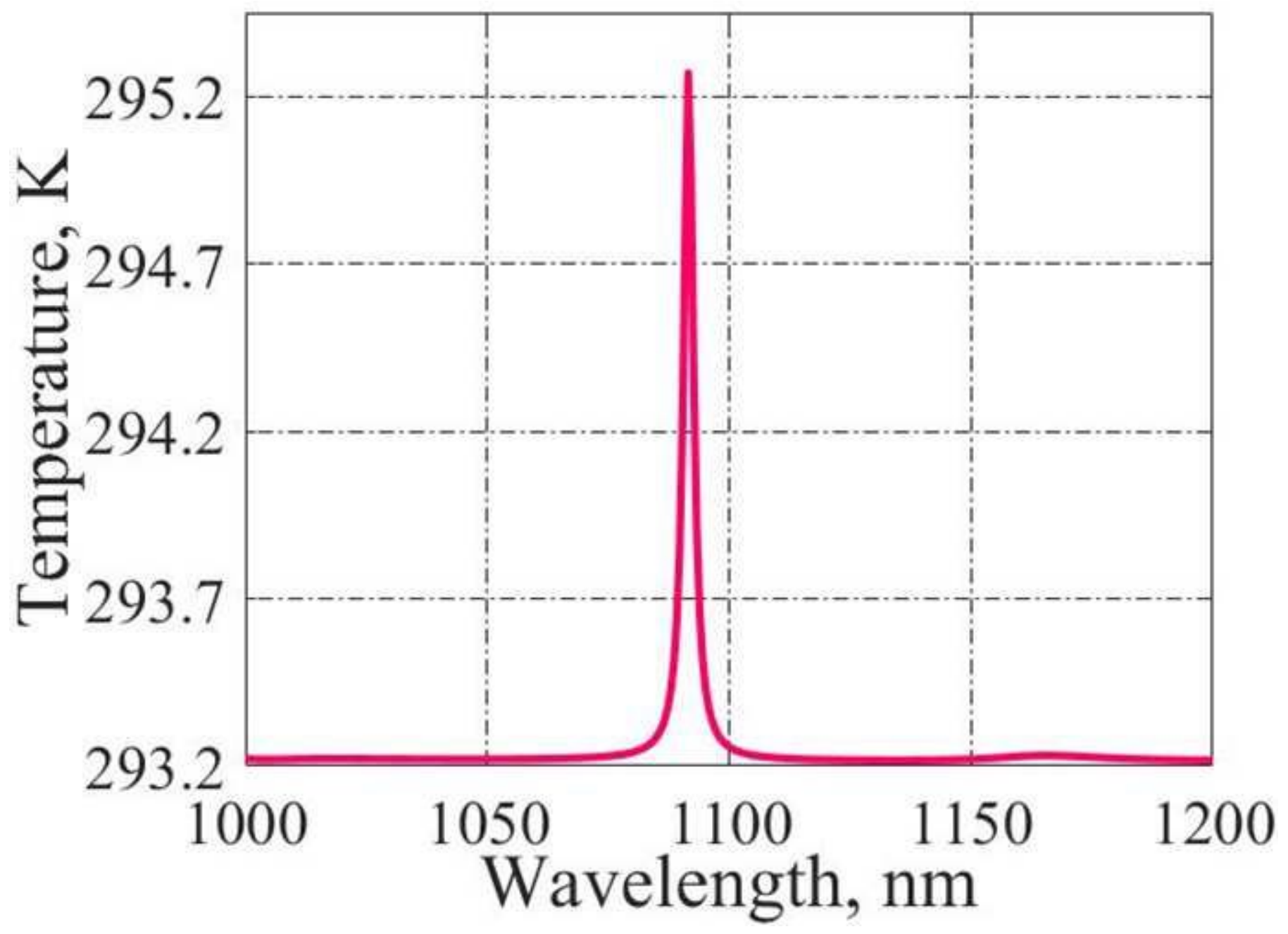

Figure 5

Maximum temperature versus the incident EM wavelength 


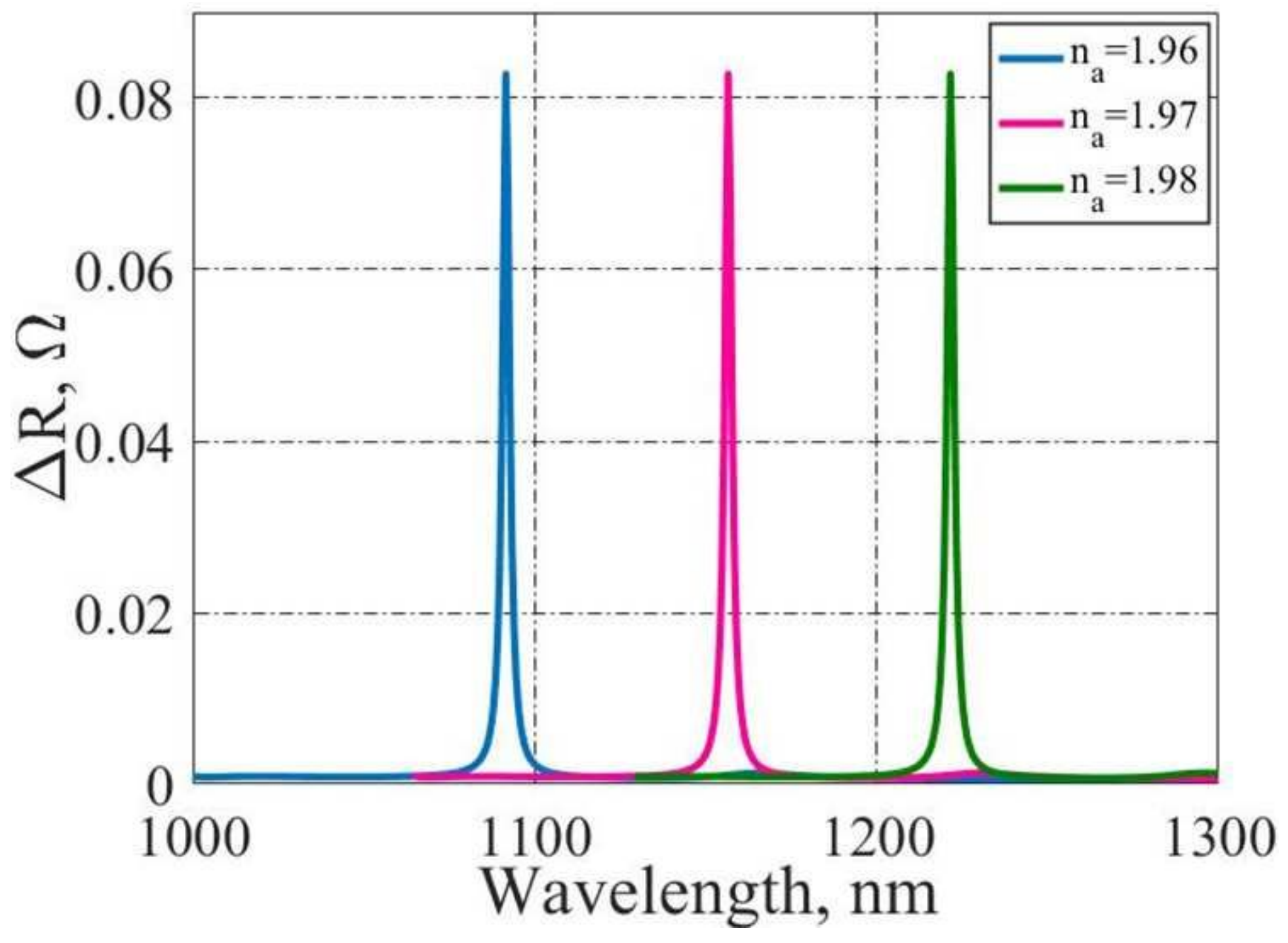

Figure 6

The spectra of the maximum $\Delta R$ versus of the incident wavelength at different refractive index of the analyte. 


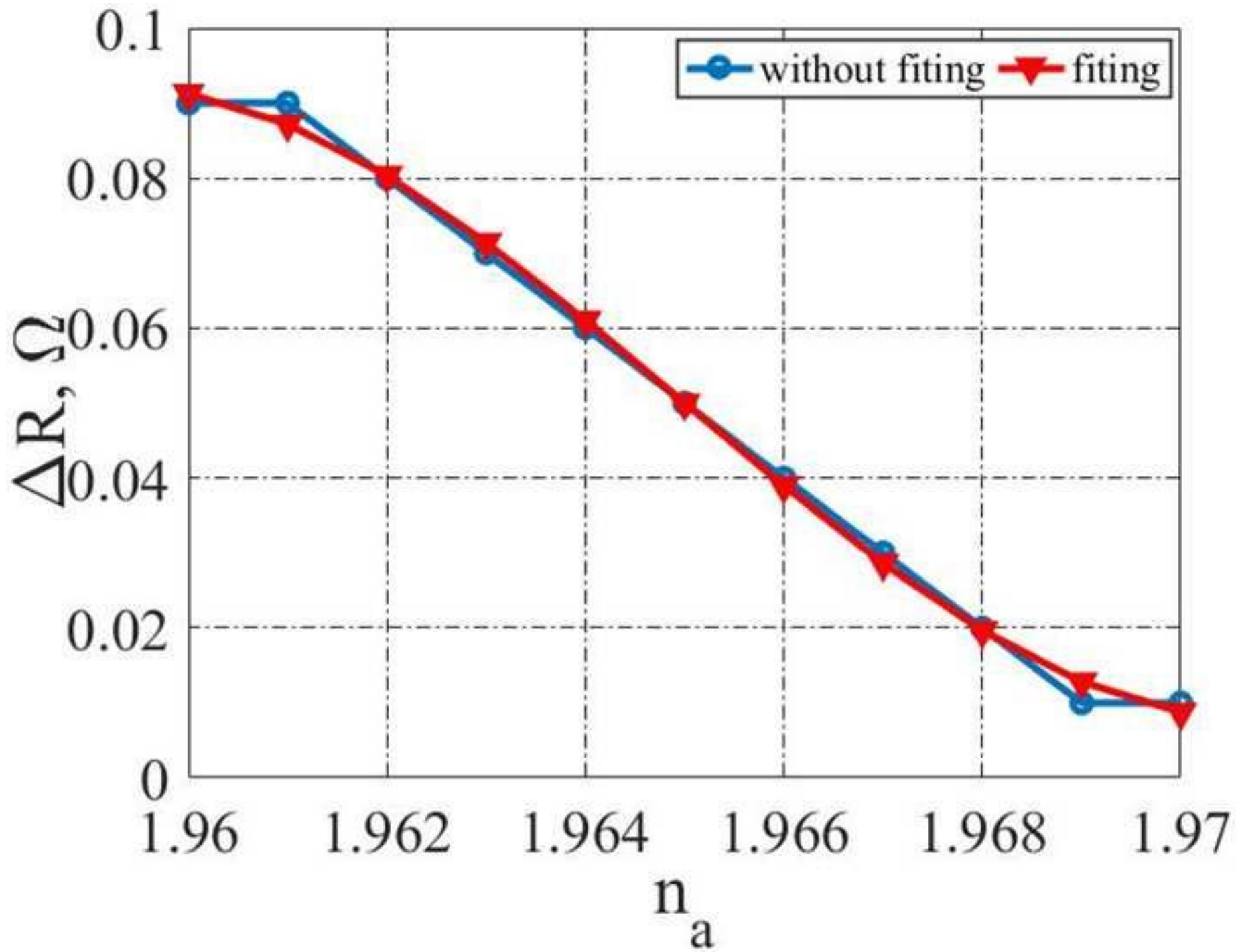

Figure 7

Variation of the resistance versus of refractive index of analyte, na, (fitting and without fitting spectra). 


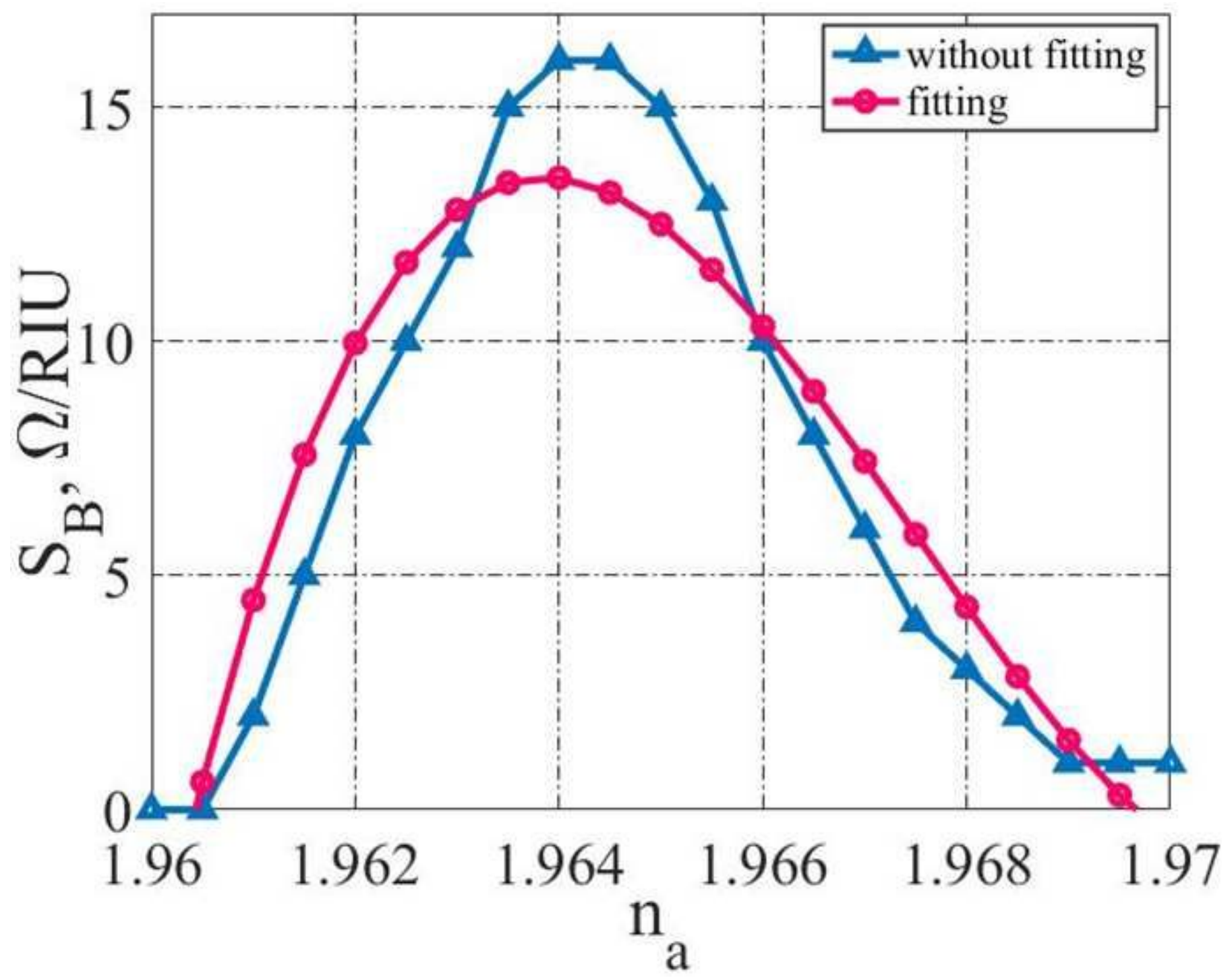

Figure 8

Sensitivity of the proposed senor versus the refractive index variations of the analyte (fitting and without fitting spectra). 


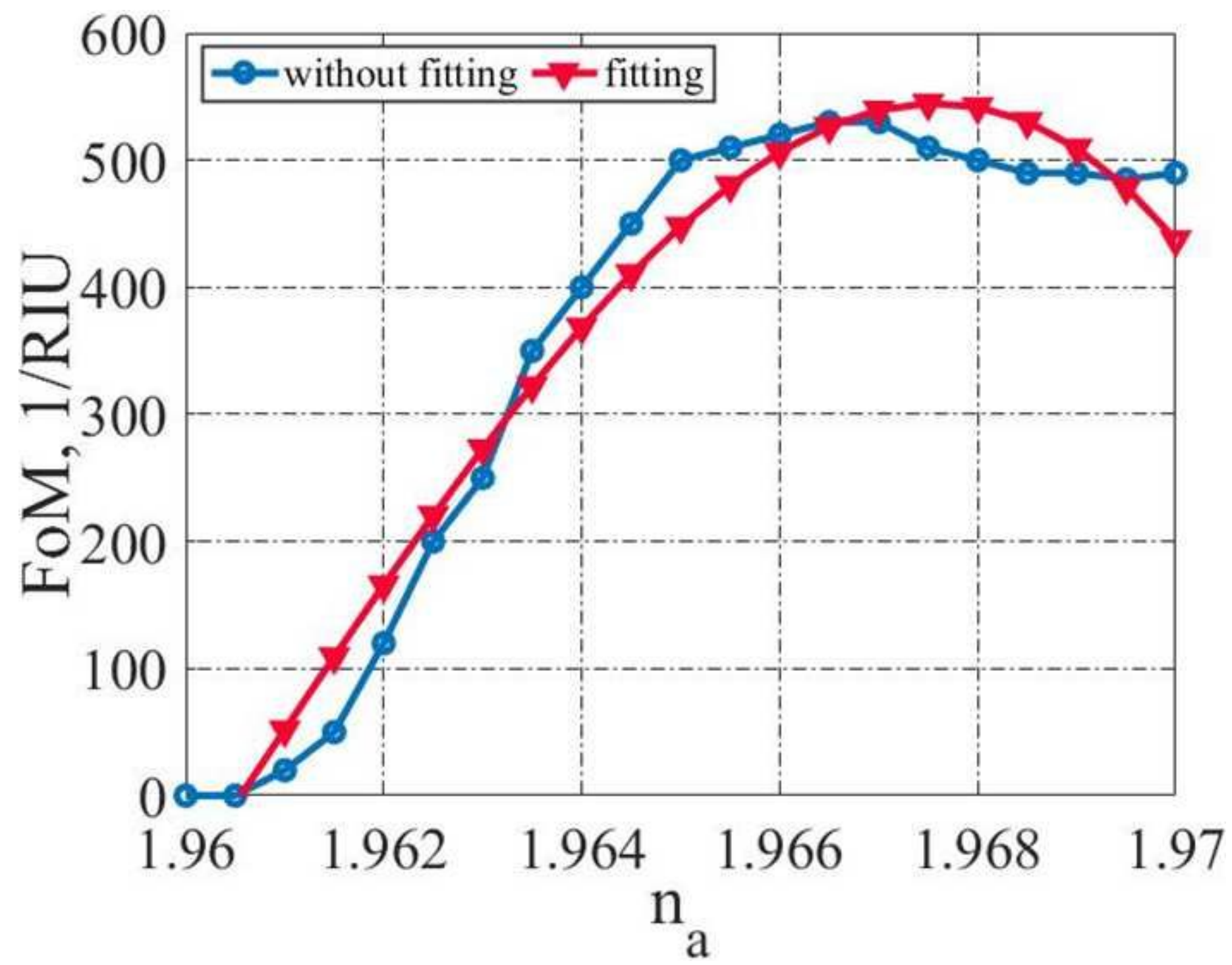

Figure 9

FoM of the proposed bolometer sensor as a function of the refractive index variation (with fitting and without fitting spectra). 